\title{
CESÀRO AVERAGE IN SHORT INTERVALS FOR GOLDBACH NUMBERS
}

\author{
ALESSANDRO LANGUASCO and ALESSANDRO ZACCAGNINI
}

\begin{abstract}
Let $\Lambda$ be the von Mangoldt function and $R(n)=\sum_{h+k=n} \Lambda(h) \Lambda(k)$. Let further $N, H$ be two integers, $N \geq 2,1 \leq H \leq N$, and assume that the Riemann Hypothesis holds. Then

$$
\begin{aligned}
\sum_{n=N-H}^{N+H} R(n)\left(1-\frac{|n-N|}{H}\right) & =H N-\frac{2}{H} \sum_{\rho} \frac{(N+H)^{\rho+2}-2 N^{\rho+2}+(N-H)^{\rho+2}}{\rho(\rho+1)(\rho+2)} \\
& +\mathcal{O}\left(N\left(\log \frac{2 N}{H}\right)^{2}+H(\log N)^{2} \log (2 H)\right),
\end{aligned}
$$
\end{abstract}

where $\rho=1 / 2+i \gamma$ runs over the non-trivial zeros of the Riemann zeta function $\zeta(s)$.

\section{INTRODUCTION}

Let $\Lambda$ be the von Mangoldt function and

$$
R(n)=\sum_{h_{1}+h_{2}=n} \Lambda\left(h_{1}\right) \Lambda\left(h_{2}\right)
$$

be the counting function for the Goldbach numbers. In this paper we are looking for an explicit formula for a Cesàro average of $R(n)$ in short intervals. Concerning long intervals, we should mention our result in [5]: assuming the Riemann Hypothesis $(\mathrm{RH})$ we have

$$
\sum_{n \leq N} R(n)=\frac{N^{2}}{2}-2 \sum_{\rho} \frac{N^{\rho+1}}{\rho(\rho+1)}+\mathcal{O}\left(N(\log N)^{3}\right),
$$

where $N$ is a large integer and $\rho=1 / 2+i \gamma$ runs over the non-trivial zeros of the Riemann zeta function $\zeta(s)$. We also mention its extension to the Cesàro average case by GoldstonYang [1] again under the assumption of $\mathrm{RH}$ :

$$
\sum_{n \leq N} R(n)\left(1-\frac{n}{N}\right)=\frac{N^{2}}{6}-2 \sum_{\rho} \frac{N^{\rho+1}}{\rho(\rho+1)(\rho+2)}+\mathcal{O}(N) .
$$

We also recall our unconditional result in [6], see also [3]: let $k>1$ be a real number; we have

$$
\begin{aligned}
\sum_{n \leq N} R(n) \frac{(1-n / N)^{k}}{\Gamma(k+1)}= & \frac{N^{2}}{\Gamma(k+3)}-2 \sum_{\rho} \frac{\Gamma(\rho)}{\Gamma(\rho+k+2)} N^{\rho+1} \\
& +\sum_{\rho_{1}} \sum_{\rho_{2}} \frac{\Gamma\left(\rho_{1}\right) \Gamma\left(\rho_{2}\right)}{\Gamma\left(\rho_{1}+\rho_{2}+k+1\right)} N^{\rho_{1}+\rho_{2}}+\mathcal{O}_{k}(N),
\end{aligned}
$$

where $\rho_{1}, \rho_{2}$ run over the non-trivial zeros of the Riemann zeta function $\zeta(s)$ and $\Gamma(s)$ is Euler's function. Our result here is

2010 Mathematics Subject Classification. Primary 11P32; Secondary 11P55.

Key words and phrases. Goldbach-type theorems, Hardy-Littlewood method. 
Theorem 1. Let $N, H$ be two integers, $N \geq 2,1 \leq H \leq N$. Assume that the Riemann Hypothesis (RH) holds. Then

$$
\begin{aligned}
\sum_{n=N-H}^{N+H} R(n)\left(1-\frac{|n-N|}{H}\right) & =H N-\frac{2}{H} \sum_{\rho} \frac{(N+H)^{\rho+2}-2 N^{\rho+2}+(N-H)^{\rho+2}}{\rho(\rho+1)(\rho+2)} \\
& +\mathcal{O}\left(N\left(\log \frac{2 N}{H}\right)^{2}+H(\log N)^{2} \log (2 H)\right),
\end{aligned}
$$

where $\rho=1 / 2+i \gamma$ runs over the non-trivial zeros of the Riemann zeta function $\zeta(s)$.

The second difference involved in the zero-depending term is natural since it depends on the symmetric nature of the short-interval Cesàro weight used in Theorem 1, Its unconditional order of magnitude is $\ll H N \exp \left(-c_{1}(\log N)^{3 / 5}(\log \log n)^{-1 / 5}\right)+N$, where $c_{1}>0$ is an absolute constant, while, under the assumption of $\mathrm{RH}$, it is $\ll H N^{1 / 2}(\log N)^{2}+N$, see Section 5 ,

In fact we will obtain Theorem 1 as a consequence of a weighted result. Letting $\psi(x)=\sum_{m \leq x} \Lambda(m)$, we have

Theorem 2. Let $N, H$ be two integers, $N \geq 2,1 \leq H \leq N$ and $y \in[-H, H]$. Assume that the Riemann Hypothesis (RH) holds. Then

$$
\max _{y \in[-H, H)}\left|\sum_{n=N-H}^{N+y} e^{-n / N}(R(n)-(2 \psi(n)-n))\left(1-\frac{|n-N|}{H}\right)\right| \ll N(\log N)^{2} \log (2 H)
$$

and

$$
\left|\sum_{n=N-H}^{N+H} e^{-n / N}(R(n)-(2 \psi(n)-n))\left(1-\frac{|n-N|}{H}\right)\right| \ll N\left(\log \frac{2 N}{H}\right)^{2} .
$$

The better estimate for the case $y=H$ depends on the second point of Lemma 5 below in which we have a more efficient estimate for the exponential sum $T_{H}(H, H ; \alpha)$, defined in (7), attached to the Cesàro weight.

For $H=N$ we can compare Theorem 11 with (11) and it is clear that the previously mentioned weakness of the available estimates for $T_{H}(H, y ; \alpha)$, again defined in (7), when $y \neq H$ leads us to a weaker final estimate by a factor $(\log N)^{3}$. Unfortunately it seems that Lemma 5 is optimal, see the remark after its proof, and hence this is a serious limitation for our method.

After being shown this paper, Goldston \& Yang told us that it should be possible to combine their technique in [1] with our Lemmas 7 and 8 below to remove the second error term in the statement of Theorem 1 .

In order to match the case $H=N$ with our method, we should have a more efficient way of removing the $e^{-n / N}$ weight (which naturally arises from the use of infinite series, see (44)); unfortunately the partial summation strategy we used to achieve this goal needs a uniform result on $y$. This leads to the first estimate in Lemma 5 and hence our global method is efficient essentially only for $H \ll N(\log \log N) /(\log N)^{3}$.

As we did in [5], we will use the original Hardy and Littlewood [2] circle method setting, i.e., the weighted exponential sum

$$
\widetilde{S}(\alpha)=\sum_{n=1}^{\infty} \Lambda(n) e^{-n / N} e(n \alpha)
$$


where $e(x)=\exp (2 \pi i x)$. Such a function was also used by Linnik [7, 8].

Acknowledgments. We thank the referee for pointing out several inaccuracies in a previous version of this paper.

\section{Setting of the Circle method}

For brevity, throughout the paper we write

$$
z=\frac{1}{N}-2 \pi i \alpha
$$

where $N$ is a large integer and $\alpha \in[-1 / 2,1 / 2]$. The first lemma is an $L^{2}$-estimate for the difference $\widetilde{S}(\alpha)-1 / z$.

Lemma 1. Assume RH. Let $N$ be a sufficiently large integer and $z$ be as in (5). For $0 \leq \xi \leq 1 / 2$, we have

$$
\int_{-\xi}^{\xi}\left|\widetilde{S}(\alpha)-\frac{1}{z}\right|^{2} \mathrm{~d} \alpha \ll N \xi(1+\log (2 N \xi))^{2}
$$

Proof. This follows immediately from the proof of Theorem 1 of [4]. We just have to pay attention to the final estimate of eq. (22) on page 315 there. A slightly more careful estimate immediately gives that (22) can be replaced by

$$
\ll \sum_{\gamma_{1}>0} \exp \left(-\frac{c}{2} \frac{\gamma_{1}}{N \eta}\right) \sum_{\gamma_{2}>0} \frac{1}{1+\left|\gamma_{1}-\gamma_{2}\right|^{2}} \ll N \eta(\log (2 N \eta))^{2} .
$$

The final estimate follows at once.

The next four lemmas do not depend on RH. By the residue theorem one can obtain

Lemma 2 (Eq. (29) of [4]). Let $N \geq 2$ and $1 \leq n \leq 2 N$ be integers; let further $z$ be as in (5). We have

$$
\int_{-\frac{1}{2}}^{\frac{1}{2}} \frac{e(-n \alpha)}{z^{2}} \mathrm{~d} \alpha=n e^{-n / N}+\mathcal{O}(1)
$$

uniformly for every $n \leq 2 N$.

Lemma 3 (Lemma 2.3 of [5]). Let $N$ be a sufficiently large integer and $z$ be as in (55). We have

$$
\int_{-\frac{1}{2}}^{\frac{1}{2}}\left|\widetilde{S}(\alpha)-\frac{1}{z}\right|^{2} \mathrm{~d} \alpha=\frac{N}{2} \log N+\mathcal{O}\left(N(\log N)^{1 / 2}\right) .
$$

Let

$$
V(\alpha)=\sum_{m=1}^{\infty} e^{-m / N} e(m \alpha)=\sum_{m=1}^{\infty} e^{-m z}=\frac{1}{e^{z}-1} .
$$

Lemma 4 (Lemma 2.4 of [5]). If $z$ satisfies (5) then $V(\alpha)=z^{-1}+\mathcal{O}(1)$.

Let now

$$
t_{H}(m)=H-|m| \text { and } T_{H}(N, y ; \alpha)=\sum_{n=N-H}^{N+y} t_{H}(n-N) e(n \alpha) .
$$


Lemma 5. Let $N, H$ be two integers, $N \geq 2,1 \leq H \leq N$. For every $y \in[-H, H)$ and $\alpha \in[-1 / 2,1 / 2]$, we have

$$
T_{H}(N, y ; \alpha) \ll H \min \left(H ; \frac{1}{\|\alpha\|}\right) .
$$

Moreover, for every $\alpha \in[-1 / 2,1 / 2]$, we also have

$$
T_{H}(N, H ; \alpha) \ll \min \left(H^{2} ; \frac{1}{\|\alpha\|^{2}}\right) .
$$

Proof. First of all we recall the well-known estimate

$$
\sum_{m=1}^{u} e(m \alpha) \ll \min \left(u ; \frac{1}{\|\alpha\|}\right) .
$$

Let now $y \in[-H, H)$. Then

$$
\left|T_{H}(N, y ; \alpha)\right| \leq \sum_{n=N-H}^{N+y} t_{H}(n-N) \ll H(H+y+1) \ll H^{2} .
$$

Moreover if $y \geq 0$ we get

$$
T_{H}(N, y ; \alpha)=H \sum_{n=N-H}^{N+y} e(n \alpha)-\sum_{m=0}^{y} m e((N+m) \alpha)-\sum_{m=1}^{H} m e((N-m) \alpha)=A-B-C,
$$

say. By partial summation and (8) we get

$$
B=y \sum_{m=0}^{y} e((N+m) \alpha)-\int_{0}^{y} \sum_{m=0}^{w} e((N+m) \alpha) \mathrm{d} w \ll \frac{y}{\|\alpha\|}+\int_{0}^{y} \frac{\mathrm{d} w}{\|\alpha\|} \ll \frac{y}{\|\alpha\|} \ll \frac{H}{\|\alpha\|} .
$$

Arguing analogously we have

$$
C \ll \frac{H}{\|\alpha\|},
$$

while the inequality $A \ll H /\|\alpha\|$ follows from (8) . If $y<0$ then we can write that

$$
T_{H}(N, y ; \alpha)=H \sum_{n=N-H}^{N+y} e(n \alpha)-\sum_{m=-y}^{H} m e((N-m) \alpha)=A-D,
$$

say, where $A$ is defined in (10). Arguing as we did for $B$ we get

$$
D \ll \frac{H}{\|\alpha\|} .
$$

Combining (91)-(13) the first part of the lemma follows for every $y \in[-H, H)$. The second part of the lemma follows by (8) and the fact that in this case we can write

$$
T_{H}(N, H ; \alpha)=\sum_{m=-H}^{H} t_{H}(m) e(m \alpha) e(N \alpha)=\left|\sum_{m=1}^{H} e(m \alpha)\right|^{2} e(N \alpha) .
$$


Remark. We remark that the estimate for $T_{H}(N, y ; \alpha), y \neq H$, is essentially optimal. For brevity, we only deal with $T_{H}(N ; y, \alpha)$ for $y \in[0, H]$. It is not hard to prove by induction that

$$
\begin{aligned}
T_{H}(N ; y, \alpha) & =e(N \alpha) \sum_{n=-H}^{y}(H-|n|) e(n \alpha) \\
& =\frac{e((N+y+1) \alpha)}{1-e(\alpha)} \cdot(y-H)+\frac{e((N+1) \alpha)}{(1-e(\alpha))^{2}} \cdot(e(y \alpha)-2+e(-H \alpha)) .
\end{aligned}
$$

In the critical range $\alpha \in\left[H^{-1}, 1 / 2\right]$ the last summand has a smaller order of magnitude than $H\|\alpha\|^{-1}$, and this implies that the bound $T_{H}(N ; y, \alpha) \ll H\|\alpha\|^{-1}$ is sharp, at least when $y \leq H / 2$, say.

We build now the zero-depending term we have in Theorem 1, The first step is the following

Lemma 6. Let $N, H$ be two integers, $N \geq 2,1 \leq H \leq N$ and $z$ be as in (5). For every $y \in[-H, H)$ we have

$$
\begin{aligned}
\int_{-1 / 2}^{1 / 2} T_{H}(N, y ;-\alpha) \frac{(\widetilde{S}(\alpha)-1 / z)}{z} \mathrm{~d} \alpha & =\sum_{n=N-H}^{N+y} e^{-n / N} t_{H}(n-N)(\psi(n)-n) \\
& +\mathcal{O}\left(H^{3 / 2} N^{1 / 2}(\log N)^{1 / 2}\right) .
\end{aligned}
$$

We remark that Lemma [6, which is a modified version of Lemma 2.5 of [5], is unconditional and hence it implies, using also Lemmas 7]-8, that the ability of detecting the zero-depending term of the Riemann zeta function $\zeta(s)$ in Theorem 1 does not depend on RH.

Proof. Writing $\widetilde{R}(\alpha)=\widetilde{S}(\alpha)-1 / z$, by Lemma 4 we have

$$
\begin{gathered}
\int_{-1 / 2}^{1 / 2} T_{H}(N, y ;-\alpha) \frac{\widetilde{R}(\alpha)}{z} \mathrm{~d} \alpha=\int_{-1 / 2}^{1 / 2} T_{H}(N, y ;-\alpha) \widetilde{R}(\alpha) V(\alpha) \mathrm{d} \alpha+\mathcal{O}\left(\int_{-1 / 2}^{1 / 2}\left|T_{H}(N, y ;-\alpha)\right||\widetilde{R}(\alpha)| \mathrm{d} \alpha\right) \\
=\int_{-1 / 2}^{1 / 2} T_{H}(N, y ;-\alpha) \widetilde{R}(\alpha) V(\alpha) \mathrm{d} \alpha+\mathcal{O}\left(\left(H^{3} N \log N\right)^{1 / 2}\right)
\end{gathered}
$$

since, by Lemmas 3 and 5 , the error term above is

$$
\ll\left(\int_{-1 / 2}^{1 / 2}\left|T_{H}(N, y ;-\alpha)\right|^{2} \mathrm{~d} \alpha\right)^{1 / 2}\left(\int_{-1 / 2}^{1 / 2}|\widetilde{R}(\alpha)|^{2} \mathrm{~d} \alpha\right)^{1 / 2} \ll\left(H^{3} N \log N\right)^{1 / 2} .
$$

Again by Lemma 4, we have

$$
\widetilde{R}(\alpha)=\widetilde{S}(\alpha)-\frac{1}{z}=\widetilde{S}(\alpha)-V(\alpha)+\mathcal{O}(1)
$$

and hence (15) implies

$$
\begin{aligned}
& \int_{-1 / 2}^{1 / 2} T_{H}(N, y ;-\alpha) \frac{\widetilde{R}(\alpha)}{z} \mathrm{~d} \alpha=\int_{-1 / 2}^{1 / 2} T_{H}(N, y ;-\alpha)(\widetilde{S}(\alpha)-V(\alpha)) V(\alpha) \mathrm{d} \alpha \\
&+\mathcal{O}\left(\int_{-1 / 2}^{1 / 2}\left|T_{H}(N, y ;-\alpha)\right||V(\alpha)| \mathrm{d} \alpha\right)+\mathcal{O}\left(\left(H^{3} N \log N\right)^{1 / 2}\right) .
\end{aligned}
$$


The Cauchy-Schwarz inequality, Lemma 5 and the Parseval theorem imply that

$$
\begin{aligned}
\int_{-1 / 2}^{1 / 2}\left|T_{H}(N, y ;-\alpha)\right||V(\alpha)| \mathrm{d} \alpha & \left.\leq\left.\left(\int_{-1 / 2}^{1 / 2} \mid T_{H}(N, y ;-\alpha)\right)\right|^{2} \mathrm{~d} \alpha\right)^{1 / 2}\left(\int_{-1 / 2}^{1 / 2}|V(\alpha)|^{2} \mathrm{~d} \alpha\right)^{1 / 2} \\
& \ll\left(H^{3} \sum_{m=1}^{\infty} e^{-2 m / N}\right)^{1 / 2} \ll\left(H^{3} N\right)^{1 / 2} .
\end{aligned}
$$

By (16)-(17), we have

$$
\begin{aligned}
\int_{-1 / 2}^{1 / 2} T_{H}(N, y ;-\alpha) \frac{\widetilde{R}(\alpha)}{z} \mathrm{~d} \alpha & =\int_{-1 / 2}^{1 / 2} T_{H}(N, y ;-\alpha)(\widetilde{S}(\alpha)-V(\alpha)) V(\alpha) \mathrm{d} \alpha \\
& +\mathcal{O}\left(\left(H^{3} N \log N\right)^{1 / 2}\right) .
\end{aligned}
$$

Now, by (44) and (6), we can write

$$
\widetilde{S}(\alpha)-V(\alpha)=\sum_{m=1}^{\infty}(\Lambda(m)-1) e^{-m / N} e(m \alpha)
$$

so that

$$
\begin{aligned}
\int_{-1 / 2}^{1 / 2} T_{H}(N, y ;-\alpha)(\widetilde{S}(\alpha)-V(\alpha)) V(\alpha) \mathrm{d} \alpha \\
\quad=\sum_{m=-H}^{y} t_{H}(m) \sum_{m_{1}=1}^{\infty}\left(\Lambda\left(m_{1}\right)-1\right) e^{-m_{1} / N} \sum_{m_{2}=1}^{\infty} e^{-m_{2} / N} \int_{-1 / 2}^{1 / 2} e\left(\left(m_{1}+m_{2}-m-N\right) \alpha\right) \mathrm{d} \alpha \\
\quad=\sum_{m=-H}^{y} t_{H}(m) \sum_{m_{1}=1}^{\infty}\left(\Lambda\left(m_{1}\right)-1\right) e^{-m_{1} / N} \sum_{m_{2}=1}^{\infty} e^{-m_{2} / N} \begin{cases}1 & \text { if } m_{1}+m_{2}=m+N \\
0 & \text { otherwise }\end{cases} \\
\quad=\sum_{m=-H}^{y} t_{H}(m) e^{-(m+N) / N} \sum_{m_{1}=1}^{m+N-1}\left(\Lambda\left(m_{1}\right)-1\right) \\
=\sum_{n=N-H}^{N+y} e^{-n / N} t_{H}(n-N)(\psi(n-1)-(n-1))
\end{aligned}
$$

since the condition $m_{1}+m_{2}=m+N$ implies that both variables are $<m+N$. Now $\psi(n)=\psi(n-1)+\Lambda(n)$, so that

$$
\sum_{n=N-H}^{N+y} e^{-n / N} t_{H}(n-N)(\psi(n-1)-(n-1))=\sum_{n=N-H}^{N+y} e^{-n / N} t_{H}(n-N)(\psi(n)-n)+\mathcal{O}(E),
$$

say, where, by the Brun-Titchmarsh theorem, we have $E \ll H^{2} \log N$ if $0 \leq H+y \leq N^{\varepsilon}$ and $E \ll H^{2}$ otherwise. By (18)-(19) and the previous equation, we have $\int_{-1 / 2}^{1 / 2} T_{H}(N, y ;-\alpha) \frac{\widetilde{R}(\alpha)}{z} \mathrm{~d} \alpha=\sum_{n=N-H}^{N+y} e^{-n / N} t_{H}(n-N)(\psi(n)-n)+\mathcal{O}\left(H^{3 / 2} N^{1 / 2}(\log N)^{1 / 2}\right)$.

Hence (14) is proved.

We need now the following lemma which is an extension of Lemma 2.6 of [5]. 
Lemma 7. Let $M>1$ be a real number. We have that

$$
\sum_{n=1}^{M}(\psi(n)-n)=-\sum_{\rho} \frac{M^{\rho+1}}{\rho(\rho+1)}+\mathcal{O}(M),
$$

where $\rho$ runs over the non-trivial zeros of the Riemann zeta function $\zeta(s)$.

Proof. The case when $M>1$ is an integer was proved in Lemma 2.6 of [5]. Let $M>1$ be a non-integral real number. Hence

$$
\sum_{n=1}^{M}(\psi(n)-n)=\sum_{n=1}^{\lfloor M\rfloor}(\psi(n)-n)=-\sum_{\rho} \frac{\lfloor M\rfloor^{\rho+1}}{\rho(\rho+1)}+\mathcal{O}(M),
$$

by Lemma 2.6 of [5]. Writing $\rho=\beta+i \gamma$, we have

$$
\frac{\lfloor M\rfloor^{\rho+1}-M^{\rho+1}}{\rho+1} \ll M^{\beta+1} \min \left(\frac{1}{M} ; \frac{1}{|\rho+1|}\right)
$$

and hence, by the zero-free region and the Riemann-von Mangoldt estimate, we obtain

$$
\sum_{\rho} \frac{\lfloor M\rfloor^{\rho+1}-M^{\rho+1}}{\rho(\rho+1)} \ll \sum_{|\rho| \leq M} \frac{M^{\beta}}{|\rho|}+\sum_{|\rho|>M} \frac{M^{\beta+1}}{|\rho|^{2}}=o(M) .
$$

By (20)-(21), Lemma 7 follows.

Lemma 8. Let $N$ be a large integer and $2 \leq H \leq N$. We have that

$$
\sum_{n=N-H}^{N+H} t_{H}(n-N)(\psi(n)-n)=-\sum_{\rho} \frac{(N+H)^{\rho+2}-2 N^{\rho+2}+(N-H)^{\rho+2}}{\rho(\rho+1)(\rho+2)}+\mathcal{O}(H N),
$$

where $\rho$ runs over the non-trivial zeros of the Riemann zeta function $\zeta(s)$.

Proof. A direct computation shows

$$
\begin{aligned}
\sum_{n=N-H}^{N+H} t_{H}(n-N)(\psi(n)-n)= & \sum_{m=0}^{H} t_{H}(m)(\psi(N+m)-(N+m)) \\
& \quad+\sum_{m=0}^{H} t_{H}(m)(\psi(N-m)-(N-m))-H(\psi(N)-N) \\
= & \sum_{m=0}^{H} t_{H}(m) a(m)-H(\psi(N)-N),
\end{aligned}
$$

where we have implicitly defined $a(m):=\psi(N+m)+\psi(N-m)-2 N$. By partial summation we have

$$
\sum_{m=0}^{H} t_{H}(m) a(m)=-H a(0)+\int_{0}^{H} \sum_{m=0}^{t} a(m) \mathrm{d} t .
$$

It is easy to see that $a(0)=2(\psi(N)-N)$ and that

$$
\sum_{m=0}^{t} a(m)=\sum_{n=N-t}^{N+t}(\psi(n)-n)+(\psi(N)-N)
$$




$$
\begin{aligned}
& =\sum_{n=1}^{N+t}(\psi(n)-n)-\sum_{n=1}^{N-t}(\psi(n)-n)+(\psi(N)-N)+^{\prime}(\psi(N-t)-(N-t)) \\
& =-\sum_{\rho} \frac{(N+t)^{\rho+1}-(N-t)^{\rho+1}}{\rho(\rho+1)}+\mathcal{O}(N),
\end{aligned}
$$

where $+^{\prime}$ indicates that the term is present only when $t$ is an integer and, in the last equality, we used Lemma 7 and the Prime Number Theorem. Summing up, exploiting the absolute convergence of the series over the zeros of the Riemann zeta function $\zeta(s)$, we obtain that

$$
\begin{aligned}
\sum_{m=0}^{H} t_{H}(m) a(m) & =-\sum_{\rho} \frac{1}{\rho(\rho+1)} \int_{0}^{H}\left((N+t)^{\rho+1}-(N-t)^{\rho+1}\right) \mathrm{d} t+\mathcal{O}(H N) \\
& =-\sum_{\rho} \frac{(N+H)^{\rho+2}-2 N^{\rho+2}+(N-H)^{\rho+2}}{\rho(\rho+1)(\rho+2)}+\mathcal{O}(H N) .
\end{aligned}
$$

Inserting (23) in (22) and using the Prime Number Theorem, Lemma 8 follows.

\section{Proof of Theorem 1}

We will get Theorem 1 as a consequence of Theorem 2, By partial summation we have

$$
\begin{aligned}
\sum_{n=N-H}^{N+H} t_{H}(n-N)(R(n)-(2 \psi(n)-n)) & =\sum_{n=N-H}^{N+H} e^{n / N} t_{H}(n-N)\left\{(R(n)-(2 \psi(n)-n)) e^{-n / N}\right\} \\
= & e^{(N+H) / N} \sum_{n=N-H}^{N+H} e^{-n / N} t_{H}(n-N)(R(n)-(2 \psi(n)-n)) \\
& -\frac{1}{N} \int_{N-H}^{N+H}\left\{\sum_{n=N-H}^{w} e^{-n / N} t_{H}(n-N)(R(n)-(2 \psi(n)-n))\right\} e^{w / N} \mathrm{~d} w+\mathcal{O}(1) .
\end{aligned}
$$

Inserting (21)-(3) in (24) we get

$$
\sum_{n=N-H}^{N+H} t_{H}(n-N)(R(n)-(2 \psi(n)-n)) \ll H N\left(\log \frac{2 N}{H}\right)^{2}+H^{2}(\log N)^{2} \log (2 H)
$$

and hence

$$
\begin{aligned}
\sum_{n=N-H}^{N+H} t_{H}(n-N) R(n) & =\sum_{n=N-H}^{N+H} t_{H}(n-N) n+2 \sum_{n=N-H}^{N+H} t_{H}(n-N)(\psi(n)-n) \\
& +\mathcal{O}\left(H N\left(\log \frac{2 N}{H}\right)^{2}+H^{2}(\log N)^{2} \log (2 H)\right) .
\end{aligned}
$$

A direct calculation proves that

$$
\sum_{n=N-H}^{N+H} t_{H}(n-N) n=H^{2} N
$$

and hence Theorem 1 now follows inserting such an identity and Lemma 8 in (25) and dividing by $H$. 
4. Proof of Theorem 2

Assume $N \geq 2,1 \leq H \leq N, y \in[-H, H]$ and let $\alpha \in[-1 / 2,1 / 2]$. Writing $\widetilde{R}(\alpha)=$ $\widetilde{S}(\alpha)-1 / z$, recalling (77) we have

$$
\begin{aligned}
& \sum_{n=N-H}^{N+y} e^{-n / N} t_{H}(n-N) R(n)=\int_{-\frac{1}{2}}^{\frac{1}{2}} \widetilde{S}(\alpha)^{2} T_{H}(N, y ;-\alpha) \mathrm{d} \alpha \\
= & \int_{-\frac{1}{2}}^{\frac{1}{2}} \frac{T_{H}(N, y ;-\alpha)}{z^{2}} \mathrm{~d} \alpha+2 \int_{-\frac{1}{2}}^{\frac{1}{2}} \frac{T_{H}(N, y ;-\alpha) \widetilde{R}(\alpha)}{z} \mathrm{~d} \alpha+\int_{-\frac{1}{2}}^{\frac{1}{2}} T_{H}(N, y ;-\alpha) \widetilde{R}(\alpha)^{2} \mathrm{~d} \alpha \\
= & I_{1}(y)+I_{2}(y)+I_{3}(y),
\end{aligned}
$$

say.

Evaluation of $I_{1}(y)$. By Lemma 2 we obtain

$$
\begin{aligned}
I_{1}(y) & =\int_{-\frac{1}{2}}^{\frac{1}{2}} \frac{T_{H}(N, y ;-\alpha)}{z^{2}} \mathrm{~d} \alpha=\sum_{n=N-H}^{N+y} t_{H}(n-N) \int_{-\frac{1}{2}}^{\frac{1}{2}} \frac{e(-n \alpha)}{z^{2}} \mathrm{~d} \alpha \\
& =\sum_{n=N-H}^{N+y} t_{H}(n-N)\left(n e^{-n / N}+\mathcal{O}(1)\right)=\sum_{n=N-H}^{N+y} e^{-n / N} t_{H}(n-N) n+\mathcal{O}(H(H+y+1)) .
\end{aligned}
$$

Estimation of $I_{2}(y)$. By (14) of Lemma 6 we obtain

$$
I_{2}(y)=2 \sum_{n=N-H}^{N+y} e^{-n / N} t_{H}(n-N)(\psi(n)-n)+\mathcal{O}\left(H^{3 / 2} N^{1 / 2}(\log N)^{1 / 2}\right) .
$$

Estimation of $I_{3}(y) ; y \in[-H, H)$. Using Lemmas 5 and 11 we have that

$$
\begin{aligned}
I_{3}(y) & \ll \int_{-\frac{1}{2}}^{\frac{1}{2}}\left|T_{H}(N, y ;-\alpha)\right||\widetilde{R}(\alpha)|^{2} \mathrm{~d} \alpha \\
& \ll H^{2} \int_{-\frac{1}{H}}^{\frac{1}{H}}|\widetilde{R}(\alpha)|^{2} \mathrm{~d} \alpha+H \int_{\frac{1}{H}}^{\frac{1}{2}} \frac{|\widetilde{R}(\alpha)|^{2}}{\alpha} \mathrm{d} \alpha+H \int_{-\frac{1}{2}}^{-\frac{1}{H}} \frac{|\widetilde{R}(\alpha)|^{2}}{|\alpha|} \mathrm{d} \alpha \\
& \ll H N\left(\log \frac{2 N}{H}\right)^{2}+H \sum_{k=0}^{\mathcal{O}(\log 2 H)} \frac{H}{2^{k}} \int_{\frac{2^{k}}{H}}^{\frac{2^{k+1}}{H}}|\widetilde{R}(\alpha)|^{2} \mathrm{~d} \alpha \\
& \ll H N\left(\log \frac{2 N}{H}\right)^{2}+H \sum_{k=0}^{\mathcal{O}(\log 2 H)} \frac{H}{2^{k}} N \frac{2^{k+1}}{H}\left(\log \frac{2^{k+2} N}{H}\right)^{2} \\
& \ll H N\left(\log \frac{2 N}{H}\right)^{2}+H N(\log N)^{2} \sum_{k=0}^{\mathcal{O}(\log 2 H)} 1 \\
& \ll H N(\log N)^{2} \log (2 H) .
\end{aligned}
$$

Estimation of $I_{3}(H)$. Using Lemmas 5 and 11 we have that

$$
I_{3}(H) \ll \int_{-\frac{1}{2}}^{\frac{1}{2}}\left|T_{H}(N, H ;-\alpha) \| \widetilde{R}(\alpha)\right|^{2} \mathrm{~d} \alpha
$$




$$
\begin{aligned}
& \ll H^{2} \int_{-\frac{1}{H}}^{\frac{1}{H}}|\widetilde{R}(\alpha)|^{2} \mathrm{~d} \alpha+\int_{\frac{1}{H}}^{\frac{1}{2}} \frac{|\widetilde{R}(\alpha)|^{2}}{\alpha^{2}} \mathrm{~d} \alpha+\int_{-\frac{1}{2}}^{-\frac{1}{H}} \frac{|\widetilde{R}(\alpha)|^{2}}{\alpha^{2}} \mathrm{~d} \alpha \\
& \ll H N\left(\log \frac{2 N}{H}\right)^{2}+\sum_{k=0}^{\mathcal{O}(\log 2 H)} \frac{H^{2}}{4^{k}} \int_{\frac{2^{k}}{H}}^{\frac{2^{k+1}}{H}}|\widetilde{R}(\alpha)|^{2} \mathrm{~d} \alpha \\
& \ll H N\left(\log \frac{2 N}{H}\right)^{2}+\sum_{k=0}^{\mathcal{O}(\log 2 H)} \frac{H^{2}}{4^{k}} N \frac{2^{k+1}}{H}\left(\log \frac{2^{k+2} N}{H}\right)^{2} \\
& \ll H N\left(\log \frac{2 N}{H}\right)^{2}+H N \sum_{k=0}^{\mathcal{O}(\log 2 H)} \frac{1}{2^{k}}\left(k+1+\log \frac{2 N}{H}\right)^{2} \\
& \ll H N\left(\log \frac{2 N}{H}\right)^{2} .
\end{aligned}
$$

End of the proof. Inserting (27)-(29) into (26), for every $y \in[-H, H)$, we immediately have

$$
\begin{aligned}
& \sum_{n=N-H}^{N+y} e^{-n / N} t_{H}(n-N) R(n)=\sum_{n=N-H}^{N+y} e^{-n / N} t_{H}(n-N) n \\
& \quad+2 \sum_{n=N-H}^{N+y} e^{-n / N} t_{H}(n-N)(\psi(n)-n)+\mathcal{O}\left(H N(\log N)^{2} \log (2 H)\right) .
\end{aligned}
$$

Hence

$$
\sum_{n=N-H}^{N+y} e^{-n / N} t_{H}(n-N)(R(n)-(2 \psi(n)-n)) \ll H N(\log N)^{2} \log (2 H)
$$

for every $y \in[-H, H)$. Thus we can write

$$
\max _{y \in[-H, H)}\left|\sum_{n=N-H}^{N+y} e^{-n / N} t_{H}(n-N)(R(n)-(2 \psi(n)-n))\right| \ll H N(\log N)^{2} \log (2 H) .
$$

The $y=H$ case follows analogously using (30) instead of (29). Dividing by $H$, Theorem 2 is proved.

\section{About the order of MAgnitude of the ZERO-DEPEnding term}

Let us define

$$
S:=\sum_{\rho} \frac{(N+H)^{\rho+2}-2 N^{\rho+2}+(N-H)^{\rho+2}}{\rho(\rho+1)(\rho+2)} .
$$

By Lemma 8 we have

$$
S=-\sum_{n=N-H}^{N+H} t_{H}(n-N)(\psi(n)-n)+\mathcal{O}(H N) .
$$

Assuming RH, we have $\psi(n)-n \ll n^{1 / 2}(\log n)^{2}$ and hence

$$
S \ll H(\log N)^{2} \sum_{n=N-H}^{N+H} n^{1 / 2}+H N \ll H(\log N)^{2}\left((N+H)^{3 / 2}-(N-H)^{3 / 2}\right)+H N
$$




$$
\ll H^{2} N^{1 / 2}(\log N)^{2}+H N .
$$

Dividing by $H$, the expected order of magnitude of the the second difference term in Theorem 1 is, under the assumption of $\mathrm{RH}, \ll H N^{1 / 2}(\log N)^{2}+N$. The same strategy works in the unconditional case too. The Prime Number Theorem in the form $\psi(n)-n \ll$ $n \exp \left(-c(\log n)^{3 / 5}(\log \log n)^{-1 / 5}\right)$, where $c>0$ is an absolute constant, leads to the final estimate

$$
\begin{aligned}
S & \ll H \exp \left(-c_{1}(\log N)^{3 / 5}(\log \log n)^{-1 / 5}\right) \sum_{n=N-H}^{N+H} n+H N \\
& \ll H^{2} N \exp \left(-c_{1}(\log N)^{3 / 5}(\log \log n)^{-1 / 5}\right)+H N,
\end{aligned}
$$

where $c_{1}>0$ is an absolute constant. Dividing by $H$, the expected order of magnitude of the the second difference term in Theorem 1 is $\ll H N \exp \left(-c_{1}(\log N)^{3 / 5}(\log \log n)^{-1 / 5}\right)+$ $N$.

\section{REFERENCES}

[1] D. A. Goldston and L. Yang. The Average Number of Goldbach Representations. arXiv.org, 2016. http://arxiv.org/abs/1601.06902.

[2] G. H. Hardy and J. E. Littlewood. Some problems of 'Partitio numerorum'; III: On the expression of a number as a sum of primes. Acta Math., 44:1-70, 1923.

[3] A. Languasco. Applications of some exponential sums on prime powers: a survey. In Proceedings of the "Terzo Incontro Italiano di Teoria dei Numeri", Scuola Normale Superiore, Pisa, 21-24 Settembre 2015, Rivista di Matematica della Università di Parma, 7: 19-37, 2016.

[4] A. Languasco and A. Perelli. On Linnik's theorem on Goldbach numbers in short intervals and related problems. Ann. Inst. Fourier, 44:307-322, 1994.

[5] A. Languasco and A. Zaccagnini. The number of Goldbach representations of an integer. Proc. Amer. Math. Soc., 140:795-804, 2012.

[6] A. Languasco and A. Zaccagnini. A Cesàro Average of Goldbach numbers. Forum Mathematicum, 27:1945-1960, 2015.

[7] Yu. V. Linnik. A new proof of the Goldbach-Vinogradow theorem. Rec. Math. [Mat. Sbornik] N.S., 19 (61):3-8, 1946. (Russian).

[8] Yu. V. Linnik. Some conditional theorems concerning the binary Goldbach problem. Izv. Akad. Nauk SSSR Ser. Mat., 16:503-520, 1952. (Russian).

Alessandro Languasco, Dipartimento di Matematica "Tullio Levi-Civita", Università di Padova, Via Trieste 63, 35121 Padova, Italy; languasco@math.unipd.it

Alessandro Zaccagnini, Dipartimento di Matematica e Informatica, Università di Parma, Parco Area delle Scienze 53/a, 43124 Parma, Italy; alessandro.zaccagnini@unipr.it 